In a number of respects the book is an improve. ment on previous bryophyte floras. The drawings of the species selected for detailed treatment are particularly valuable; and include habit drawings as well as figures of details important for identification. The descriptions are straightforward but sufficiently full, and are accompanied by useful ecological notes. Habitat lists at the end of the book indicate the range of species most likely to be encountered in twenty-five different habitats, and should be of great value to those beginning field-studies of the bryophytes. Mention should also be made of the fine photographs, which illustrate the attractive appearance of some of the common mosses and liverworts.

It may be said in conclusion that Dr. Watson. has succeeded admirably in his aim of providing a sound introductory flora of the British bryophytes. The British student is now well provided with floristic works; but there is still a great need for an introductory work dealing with the more general aspects of bryology. Anyone acquainted with the classical works of Hofmeister, Leitgeb, Goebel and others, and with the many recent contributions to the morphology, physiology, cytology, genetics and general biology of the bryophytes, will realize that taxonomic studies are only one approach to the many aspects of bryological science.

A. Allsopp

\section{BLACK FLIES OF GUATEMALA}

The Black Flies (Diptera, Simuliidae) of Guatemala and their Role as Vectors of Onchocerciasis

By Herbert T. Dalmat. (Smithsonian Miscellaneous Collections, Vol. 125, No. 1, Pub. 4173.) Pp. $425+$ $44 \mathrm{pl}$. (Washington, D.C. : Smithsonian Institution, 1955.)

IN the New World onchocerciasis is endemic in 1 certain areas in Guatemala, Mexico, elsewhere in Central America, and in Venezuela. In communities within these areas in Guatemala, the degree of infection may vary from 5 to 100 per cent, with an average of about 35 per cent. About half those infected suffer from ocular symptorns, and about 5 per cent suffer the most serious sequela, namely, blindness. It has been estimated that some thirtyfive thousand persons in Mexico and some twenty-five thousand in Guatemala are infected. As in Africa, the infecting worms are transmitted from man to man by species of Simuliidae. Nuisance caused by the biting flies can also bring to a halt field-operations, particularly the harvesting of coffee, which is the most important crop in the endemic areas of Guatemala. Prior to 1934 only three species of Simuliidae were known from Guatemala; that year Bequaert ${ }^{1}$ added three more. To anyone acquainted with the Simuliidae and able to read maps, this was obviously a quite unrealistic picture of the Simuliid fauna in a country like Guatemala. This book by H. T. Dalmat presents the results of nearly six years (1947-53) of intensive work on the Simuliidae in Guatemala, done with the support of various agencies and the Ministry of Health there. Forty-one species are dealt with and, of these, thirteen are ones that Dalmat has described as new in the course of his studies in Guatemala in earlier papers.

The various species are described in detail : both sexes, the pupa with its cocoon, and the mature larva when, as in most cases, diligent field-work or controlled rearing has provided them. No new species are described; but there are descriptions of sexes and stages not previously known. Keys for identification of the species in all stages are provided, and there are excellent series of figures of genitalia, etc., all drawn in the same style from uniformly prepared parts. Uniformity of style and preparation is important because parts such as the male claspers can give very different impressions of their characteristics if snipped off the abdomen at slightly different angles. The keys are restricted to the species known from Guatemala, and one therefore wonders if there is much real value in giving a key to the sub-genera of Simulium. All that this key does is to pick out three species which are the only representatives of their different sub-genera in Guatemala and then lead on to other keys to the remaining species of the other four sub-genera represented in the Guatemalan fauna. Only the more important and more recent references for each species are given, and the reader is referred to Vargas's ${ }^{2}$ catalogue of "New World Simuliidae" for the complete literature up to the date of that catalogue (1945). The status of genera and sub-genera is not discussed.

Valuable and important as these systematic descriptions are, the parts of the book that will interest Old World readers most are probably those dealing with epidemiology (pp. 6-57) and ecology (pp. 313-372). Anyone proposing to carry out a survey of Simuliidae would do well to study Dalmat's approach and methods. The various tables, graphs, etc., obviously represent but a creaming of a vast amount of data collected.

In certain regions the rainy season is inimical to Simuliidae because the exceptional amounts of water scour large numbers of larvæ and pupæ from their substrates and wash them down-stream to conditions under which, even if they could gain a foothold, they would not survive. The coffee crop is usually concentrated in small patches cleared in the forest. At harvest large numbers of workers go to these coffee fields, and men especially will expose the upper parts of the body and roll up their trouser legs when picking the berries. Furthermore, while picking berries, the workers stand relatively still in one place and so present excellent targets for the flies. If the harvest coincides with a good emergence of adult Simuliidae, then conditions are ideal for spreading the infection of onchocerciasis, for the adult flies will travel considerable distances to obtain a blood. meal.

Only three species of Simulium appear to be of importance as vectors of onchocerciasis in Guatemala: ochraceum Walker, metallicum Bellardi and callidum Dyar and Shannon. These three are markedly anthropophilic in habit compared with the remaining species. Itinerant peddlers are a feature of Guatemalan village life, and they may possibly be a source of infection. The exact factors that control endemicity are not yet apparent: the principal vector seems to be $S$. ochraceum Walker; but the areas of endemicity do not extend to the margins of the distribution of this species.

The Smithsonian Institution is to be congratulated on the general format and production of this book, in which it has been assisted financially by the PanAmerican Sanitary Bureau. JoHN SMart

${ }^{1}$ Bequaert, J. C., in Strong, R. P., Sandground, J. D., Bequaert, J. C. and Ochoa, M. M., "Onchocerciasis, with special Reference to the Central American Form or the Disease (Dept. Trop. Med. and Inst. Trop. Biol. Med., Harvard Univ., Contr. No. 6, 234; 1934) 2 Vargas, L., Inst. Salubr. Enferm. Trop., Mexico, No. 1 (1945). 